\title{
PERSISTENT GENITAL AROUSAL DISORDER INTERVENTION WITH MANUAL PHYSIOTHERAPY - A CASE STUDY
}

\author{
MARIYAM FARZANA SF*, VEERA GOUDHAMAN TS, PAWAN KUMAR MS
}

RAA Physiotherapy Clinic, Chennai, Tamil Nadu, India. Email: mariyamfarzana86@yahoo.com

Received: 17 May 2018, Revised and Accepted: 27 June 2018

ABSTRACT

A 36-year-old female patient came with an bilateral lower extremity pain and altered sensation, physical evaluation and radiological finding are normal, and there was presence of myofascial trigger points in both lower extremities diagnosed as a myofascial pain syndrome (MPS) and had been treated for it. The failure of treatment for MPS is followed by reevaluation, and on subjective evaluation, her genital symptoms such as unresolving genital arousal, fullness, engorgement, and restless leg syndrome were revealed, and on the objective evaluation, there was an overactive pelvic floor muscles, with subjective and objective findings, the problem was diagnosed as Persistent Genital Arousal Disorder (PGAD), the problem causing pathoanatomic structure and pathomechanism was discovered and addressed with manual physiotherapy, and the patient got complete solution from the PGAD. The informed consent was obtained from the patient.

Keywords: Persistent genital arousal disorder, Myofascial trigger points, Genital arousal, Restless leg syndrome, Overactive pelvic floor muscle, Manual physiotherapy.

(C) 2018 The Authors. Published by Innovare Academic Sciences Pvt Ltd. This is an open access article under the CC BY license (http://creativecommons. org/licenses/by/4. 0/) DOI: http://dx.doi.org/10.22159/ajpcr.2018.v11i10.27327

\section{INTRODUCTION}

Persistent genital arousal disorder (PGAD) is a sudden, intrusive, and undesirable arousal of the genitals without any desire or interest in the sex [1]. Leiblum and Nathan in 2001 were credited to describe this disorder first [2]. PGAD is clinically manifested by altered tingling, throbbing, and pulsatile sensation in genitals along with arousal of genitals and engorgement, these symptoms commonly present in the clitoris, vagina, and labia [1]. These symptoms commonly persist for hours, days, and even for weeks, and these sensation do not resolve even after orgasm. The PGAD accompanies with overactive bladder, restless leg syndrome (RLS) less oftenly, and restless genital syndrome (RGS) oftenly [1]. Etiology of the PGAD - Psychological factors such as sex-related emotional problems, anxiety disorder, depressive disorder, and sex-abuse [3], Physiological factors - central mechanisms such as central dopaminergic dysfunction and hormonal [2,3], Pelvic vascular malformation [3], Mechanical - overactive pelvic floor muscles [1], Dietary - phytoestrogens like soy, phyto-estrogens are phytochemicals present in plants are known for their anticancer, antioxidant, immunitypotentiating, neuropharma-cological and detoxifying functions $[1,3,4]$, Medications - such as serotonin [1,3]. Pathogenisis - The neuropathogenisis of PGAD hypothized as stress, drugs, substances, polyneuropathy, menopause, pelvic vascular anomaly, and mechanical irritations affect pudendal nerve and dorsal clistoral nerve, and the product of this will be PGAD along with the RLS/RGS and overactive bladder [1,3]. Stress, drugs, substances, polyneuropathy, menopause, pelvic vascular anomaly, and mechanical irritations affect pudendal nerve and dorsal clistoral nerve, and the product of this will be PGAD along with the RLS/RGS and overactive bladder [1,3]. Diagnostic criteria of PGAD [3] are as follows: (a) Sudden genital arousal that persists for prolonged periods of time (hours, days, and months), (b) persistent arousal which not gets into resolution with orgasm, (c) the sexual desire will never be accompanied with arousal, (d) arousal that will be unwished one, and (e) the symptoms often cause distress in the patient.

\section{CASE REPORT}

A 36-year-old female patient after consultations with gynaecologist, urologist, orthopaedic surgeon, and general practitioner, came with the complaint of bilateral lower extremity diffused pain and altered sensations during the sleep and rest. On subjective evaluation, there was no altered sensation or pain in both the lower extremities of the patient. On physical evaluation, neurological and orthopedical problems are ruled out with neuromusculoskeletal evaluation, there was somatic dysfunction of lumbopelvic and hip, On manual muscle testing (MMT) the power of muscles in lower extremities was grade 3 and $<$ grade 3 , there was presence of diastasis rectus abdominus, radiological findings revealed no significant abnormalities on spine and pelvis. On palpation, there was diffused tenderness in bilateral lower extremities with myofascial trigger points, diagnosed as myofascial pain syndrome and addressed her with trigger point deactivation, and even after 10 sessions, there is no significant improvement in pain and altered sensation while sleeping and on rest. After $10^{\text {th }}$ session, the patient was reexamined, with subjective evaluation, the patient disclosed about her genital arousal, altered sensations in genitals, and awkward posture which she assumes while sitting and faulty body mechanics while walking. On physical evaluation, pelvic floor muscles have been evaluated, the evaluation revealed pelvic floor dysfunction (overactive pelvic floor), and there was presence of myofascial trigger points on the levator ani, obturator internus, and adductor group of muscles. On the deactivation of trigger points in the levator ani, obturator internus, and adductor muscles, the patient symptoms had started to resolve gradually from the fourth session of treatment, and on the $11^{\text {th }}$ session of treatment, there was a complete resolution.

\section{DISCUSSION}

The inconclusive diagnosis of the problem on the first subjective and objective examination was concluded on second subjective and objective examination. The diagnosis is made as PGAD. Based on the verbal confession with the patient and disclosure of her genital symptoms on subjective evaluation and pelvic floor evaluation. These patient symptoms were spontaneous genital arousal, genital fullness, engorgement, RGS, RLS, and overactive bladder. In PGAD, there will overactivity of pelvic floor anterior-most muscles [3]. The classical symptoms are caused by vasocongestion and pudendal nerve compression [3]. The overactive pelvic floor muscles and obturator internus compress the pudendal vein, as a consequence there will be blockage of venous outflow, and it 
manifests the clinical symptoms [3]. The compression of pudendal nerve causes altered sensation and pain on its distributing areas. The pudendal nerve is an mixed nerve (motor and sensory), and it accompanies the pudendal artery and vein throughout its distribution [5]. How this patient got PGAD? How deactivation of myofascial trigger points in levator ani, obturator internus, and adductor muscles relieved her from the PGAD? What was the pathomechanism? And what was the pathoanatomical structure? Hypothesizing of these things is intention of this paper. The patient had a habit of maintaining her lower extremity in external rotation from the hip while standing and walking, she use to sit with one leg abducted position while sitting in the floor, and while doing household activities. Obturator internus muscle along with piriformis acts as an hip external rotator on standing and it acts as a hip abductor while hip is flexed [6], commonly it acts along with other hip external rotators, and all the hip external rotators act together in similar fashion. While the hip is flexed, the lateral rotators of hips act as hip abductors and become as internal rotators rather than the external rotator [7], this situation creates an overtaxing of the muscles, and as a consequence, it harbors trigger points. In this patient while standing and walking, the obturator internus works more along with other hip external rotators, and during sitting, the obturator internus again works more along with other hip external rotators as abductors and internal rotators. The sustained contraction of the muscle due to faulty posture will cause trigger points in obturator internus due to overtaxing and adductor muscles due to stretched postion, as consequence, the muscles will harbor trigger points, and due to presence of the myofascial trigger points, there will be tightness of these muscles [8]. This faulty posture has profound influence on the pelvic girdle structures; as a consequence, there will be overactivity of pelvic floor muscles. Few researchers had described that the trigger points in piriformis can contribute associated trigger points in levator ani $[8,9]$, and according to Gray's Anatomy, levator ani originates from the line around the pelvic wall as a condensation of obturator internus fascia [10], the piriformis is an primary external rotator of femur along with the obturator internus and other external rotator, so involvement of piriformis with obturator internus will be there and harborization of trigger points due to overtaxing will occur. Hence, the faulty posture with overtaxing external rotators of femur contributes associated trigger points on levator ani, and as a consequence, there will be overactive pelvic floor muscle. Though with the existence of faulty posture, faulty body mechanics, overactive pelvic floor and etc. how mere de-activating the myofascial trigger points in levator ani, obturator internus and adductor muscles alone resolved the symptom? The Alcock canal is formed by the obturator internus fascia [11], and this Alcock canal contains the internal pudendal nerve, artery, and vein. Hence, the tightness of the obturator internus due to trigger points compresses the contents of Alcock canal [8]. Hence, deactivation of trigger points lengthened the muscle and relieved the compression, and as a consequence, the symptoms of PGAD relieved. The hands-on treatment protocol used in this patient was trigger point deactivation with trigger point release technique per vaginal [12] and soft tissue technique for obturator internus [13], cross friction massage on peripeheral musculature of bilateral lower extremeties, stretching of obturator internus and adductor muscles,which is similar to Upavista Konasana which stretches the inner thighs, hips and groin; relaxes the pelvic-floor muscles [14].

\section{CONCLUSION}

The PGAD, a unreported new condition affecting females, though few researchers is not in clear stance on the grounds of its etiology, and there are some researchers hypothesized the presence of mechanical causes. This paperwork with single-case study is intended to hypothesize that improper postures, myofascial trigger points in the muscles of pelvic floor, lateral rotators of femur, and adductors of femur can cause PGAD. The above-discussed pathomechanism and pathoanatomic structure can solely cause PGAD and addressing these structure can resolve PGAD, but it needs more study with more samples to validate this mechanical cause and the above-discussed treatment protocol.

\section{CONFLICTS OF INTEREST}

Authors do not have any conflicts of interest.

\section{CONTRIBUTION BY AUTHORS}

S.F. Mariyam Farzana PT: Introduction, etiology, diagnostic criteria, treatment application, discussion, and conclusion, T.S. Veera Gaudhaman PT: References, citations, and plagiarism checking, M.S. Pawan Kumar PT: Discussion, conclusion, and conceptualization of the study.

\section{REFERENCES}

1. Padoa A, Rosenbaum TY. The Overactive Pelvic Floor. Switzerland: Springer; 2016.

2. Nazik H, Api M, Aytan H, Narin R.A new medical treatment with botulinum toxin in persistent genital arousal disorder: Successful treatment of two cases. J Sex Marital Ther 2014;2014:1-5

3. Hrynko M, Kotas R, Pokryszko-Dragan A, Nowakowska-Kotas M, Podemski R. Persistent genital arousal disorder-a case report. Psychiatr Pol 2017;51:117-24.

4. Prasad SM, Venugopal SP, Alagarsamy V, Sridevi C. The preliminary phytochemicals analysis and oral acute toxicity study of stem bark. Int J Pharm Pharm Sci 2016;8:975-1491.

5. Barrel JP, Crobber A. Viscero-Vascular Manipulation. Philadelphia, PA: Churchill Livingston;2011. Sebastian D.

6. Sebastian D. Principles of Manual Therapy. Elsevier Saunders: Jaypee Publications; 2008.

7. Neumann DA. Kinesiology of the hip: A focus on muscular actions. J Orthop Sports Phys Ther 2010;40:82-94.

8. Travell JG, Simons DG. Myofascial Pain and Dysfunction. The Trigger Point Manual, The Lower Extremeties. Vol. 2. Baltimore: Lippincott Williams and Wilkins; 1984.

9. Pace JB, Nagle D. Piriform syndrome. West J Med 1976;124:435-9.

10. Smith CF, Dilley A, Mitchell BS, Drake RL. Gray's Surface Anatomy and Ultrasound. A Foundation for Clinical Practice. Elsevier Saunders: Elsevier; 2018

11. Rea W, Curran N. Abdominal pelvic pain syndromes. Contin Educ Anaesth Crit Care Pain 2015;1 38-43 5

12. Faubion SS, Shuster LT, Bharucha AE. Recognition and management of nonrelaxing pelvic floor dysfunction. Mayo Clin Proc 2012;87:187-93.

13. Stone C. Visceral and Obstetrics Osteopathy. United Kingdom: Churchill Livingstone Elsevier; 2007

14. Sengupta $P$. The bliss yoga inculcates during the different stages of pregnancy. Int J Pharm Pharm Sci 2014;6:975-1491. 